Preprint typeset using $\mathrm{LAT}_{\mathrm{E}} \mathrm{X}$ style emulateapj v. 5/2/11

\title{
DISCOVERY OF AN APPARENT HIGH LATITUDE GALACTIC SUPERNOVA REMNANT
}

Robert A. Fesen, Jack M. M. Neustadt, Christine S. Black, \& Ari H. D. Koeppel

6127 Wilder Lab, Department of Physics \& Astronomy, Dartmouth College, Hanover, NH 03755 USA

\section{ABSTRACT}

Deep $\mathrm{H} \alpha$ images of a faint emission complex $4.0^{\circ} \times 5.5^{\circ}$ in angular extent and located far off the Galactic plane at $l=70.0^{\circ}, b=-21.5^{\circ}$ reveal numerous thin filaments suggestive of a supernova remnant's shock emission. Low dispersion optical spectra covering the wavelength range 4500 - 7500 $\AA$ show only Balmer line emissions for one filament while three others show a Balmer dominated spectrum along with weak [N I] 5198, $5200 \AA$, [O I] 6300, $6364 \AA$, [N II] $6583 \AA$, [S II] 6716, 6731 and in one case [O III] $5007 \AA$ line emission. Many of the brighter $\mathrm{H} \alpha$ filaments are visible in near UV GALEX images presumably due to C III] 1909 A line emission. ROSAT All Sky Survey images of this region show a faint crescent shaped X-ray emission nebula coincident with the portion of the $\mathrm{H} \alpha$ nebulosity closest to the Galactic plane. The presence of long, thin Balmer dominated emission filaments with associated UV emission and coincident X-ray emission suggests this nebula is a high latitude Galactic supernova remnant despite a lack of known associated nonthermal radio emission. Relative line intensities of the optical lines in some filaments differ from commonly observed $[\mathrm{S} \mathrm{II}] / \mathrm{H} \alpha$ $\geq 0.4$ radiative shocked filaments and typical Balmer filaments in supernova remnants. We discuss possible causes for the unusual optical SNR spectra.

Subject headings: ISM: individual objects: G70.0-21.5, ISM: supernova remnant - shock waves - optical - X-rays: ISM

\section{INTRODUCTION}

Currently, there are 294 confirmed and nearly two dozen possible or suspected Galactic supernova remnants (SNRs) (Green 2014a). The majority of these were first identified in the radio due to nonthermal radio emissions associated with shocked gas (Milne 1970; Downes 1971). Roughly $40 \%$ of the known Galactic SNRs exhibit associated X-ray emission with a smaller percentage $(\sim 30 \%)$ showing some coincident optical emission.

New Galactic SNRs are still occasionally identified in the radio (de Gasperin et al. 2014; Gao \& Han 2014; Gerbrandt et al. 2014) and X-rays (Renaud et al. 2010; Reynolds et al. 2013). Although discoveries of Galactic remnants in the optical are relatively rare, several have recently been made (e.g.. Stupar et al. 2008; Fesen \& Milisavlievic 2010; Sabin et al. 2013) through deep $\mathrm{H} \alpha$ surveys such as the $\mathrm{H} \alpha$ emission Virginia Tech Spectral Line Survey (VTSS) of the Galactic Plane (Dennison et al. 1998; Finkbeiner 2003) and the Isaac Newton Telescope Photometric $\mathrm{H} \alpha$ Survey (Drew et al. 2005; González-Solares et al. 2008).

Here we report the discovery of an apparent Galactic SNR located well off the Galactic plane. The remnant consists of faint emission covering a region some $4.0^{\circ} \times 5.5^{\circ}$ in angular size and spherical in shape along its eastern boundary. Deep $\mathrm{H} \alpha$ imaging and optical spectroscopic observations of portions of this emission complex are described in $\S 2$ with the results presented and discussed in $\S 3$.

\section{OBSERVATIONS}

Examination of VTSS $\mathrm{H} \alpha$ images revealed a large, faint, and relatively isolated optical emission nebulosity located well off the galactic plane. Figure 11 shows this nebula in the VTSS $\mathrm{H} \alpha$ survey. The nebulosity is roughly $4.0^{\circ} \times 5.5^{\circ}$ in angular extent and centered ap- proximately at $l=70.0^{\circ}, b=-21.5^{\circ}$ corresponding to $\mathrm{RA}=21^{\mathrm{h}} 24^{\mathrm{m}}$, Dec $=+19^{\circ} 23^{\prime}(\mathrm{J} 2000)$.

This emission is not part of a cataloged galactic nebula or H II region (Sharpless 1959; Maršálková 1974; Neckel \& Vehrenberg 1985). It is also too faint to be seen in the $\mathrm{H} \alpha$ survey by Sivan (1974) and lies too far off the plane to be covered in the optical emission line survey of the Milky Way by Parker et al. (1979). However, some of its brighter regions are faintly visible on both first and second digital Palomar Observatory Sky Survey (POSS) red images.

The nebula, denoted G70.0-21.5 and hereafter also referred to as G70, exhibits an irregular morphology but is roughly circular along its eastern boundary. While its extent in the west is uncertain due to missing VTSS data to the southwest and possible confusion with fainter neighboring emission features to the west, G70's angular size is at least $4.0^{\circ} \times 5.5^{\circ}$ based on its appearance on the VTSS images and our follow-up $\mathrm{H} \alpha$ images described below.

Nine regions of this nebulosity were imaged in June and October 2014 at the MDM Observatory at Kitt Peak (see Fig. 2). The images were obtained using a backside illuminated $2048 \times 2048$ SITe CCD detector and $30 \AA$ and $90 \AA$ FWHM H $\alpha$ filters. Regions 1 through 5 were imaged using the $2.4 \mathrm{~m}$ Hiltner telescope which provided a $9^{\prime}$ field of view with an image scale of 0.275 pixel $^{-1}$. Regions 6 through 9 were imaged on the $1.3 \mathrm{~m}$ McGrawHill telescope producing a field of view of approximately $17^{\prime}$ with an image scale of $0^{\prime \prime} .508$ pixel $^{-1}$. Table 1 lists the coordinate centers and exposure times for all nine regions.

Low-dispersion optical spectra of filamentary features in this nebula were obtained in June 2014 using the MDM $2.4 \mathrm{~m}$ telescope with the Mark III Spectrograph and a $1024 \times 1024$ Tektronix CCD detector. A $1^{\prime \prime} .2 \times 4.5^{\prime}$ slit 
and a 300 lines $\mathrm{mm}^{-1} 5400 \AA$ blaze grism were used to obtain sets of two or three $1000-1200$ s exposures spanning the spectral region $4500-7500 \AA$ with a spectral resolution of $8 \AA$.

Standard pipeline data reduction of these images and spectra was performed using IRAFI. This included debiasing, flat-fielding using twilight sky flats, and dark frame corrections. Spectral data were reduced using standard IRAF software routines and calibrated with $\mathrm{Hg}, \mathrm{Ne}$, and Xe lamps and Oke (1974) Massey \& Gronwald (1990) standard stars. Flat fielding of the images did not always result in flat background intensity levels for some of the $2.4 \mathrm{~m}$ images, most apparent along the edges of the images for Regions 1 and 2 .

\section{RESULTS AND DISCUSSION}

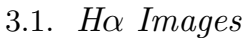

Although appearing diffuse in the low resolution (1.6 pixel $^{-1}$ ) VTSS images with only a hint of filamentary structure from its relatively sharp eastern edge, our $\mathrm{H} \alpha$ images of nine regions of this nebulosity (Fig. 22) revealed dozens of thin emission filaments (Figs. $3-5$ ). The $\mathrm{H} \alpha$ filaments in Regions 1,2, 3 and 5 along the nebula's eastern and southern edges indicate a definite boundary to the emission complex in these areas. The presence of long, thin filaments in the western Regions 6 through 9 , like those detected in the east, suggests the nebula extends at least out to these locations.

Faint and unresolved filaments appear throughout much of the G70 nebula, forming a complex of overlapping filamentary structures in places, often curved, and extending many arcminutes in length. An example is in Region 3 (Fig. 5) where a long continuous emission filament lies along the southern border of the nebula.

The close spacing and alignment of filaments, as seen in Regions $1,2,6$, and 7 , is suggestive of a thin expanding emission shell being viewed nearly edge on where small velocity differences across its surface create the appearance of close or overlapping filaments. Consistent with this picture, faint diffuse trailing emission can be seen associated with many filaments.

While much of G70's nebulosity is filamentary, it also contains patches of diffuse emission, especially along its western boundary. Our image of Region 9 shows considerable diffuse emission which extends to the northwestern edge of the image frame, and an exploratory image taken farther north showed only a broad band of diffuse emission.

\subsection{Optical Spectra}

Low dispersion spectra were taken of filaments in Regions 3 and 5 with the slit positions shown in Figure 6 The resulting spectra are presented in Figure 7

For Region 3, spectra were extracted for a faint, more westerly filament (Filament 1) and for a brighter, curved filament (Filament 2) farther to the east. For Region 5, we extracted spectra for a small sharp filament (Filament 3 ) and combined a $20^{\prime \prime}$ long region of emission behind this filament into one spectrum (Filament 4).

${ }^{1}$ IRAF is distributed by the National Optical Astronomy Observatories, which is operated by the Association of Universities for Research in Astronomy, Inc. (AURA) under cooperative agreement with the National Science Foundation.
Filament 1 in Region 3 was found to exhibit a Balmer dominated emission spectrum. Only $\mathrm{H} \alpha$ emission was clearly detected $(\mathrm{H} \beta$ was present but barely above the noise level) with no hint of [N II] $6583 \AA$ or [S II] 6716, $6731 \AA$ line emissions down to a level below $10 \%$ of the strength of $\mathrm{H} \alpha$. This Balmer dominated type spectrum could be seen extending some $15^{\prime \prime}$ behind (eastward) of this filament.

In contrast, a very different spectrum was seen for the much brighter filament marked Filament 2 in Region 3 (see Fig. 6i). While this filament also showed a Balmer dominated spectrum, it exhibited faint emission lines of [N I] 5198, $5200 \AA$, [O I] 6300, $6364 \AA$, [N II] $6583 \AA$, and [S II] $6716,6731 \AA$. The change in spectra between Filaments 1 and 2 was abrupt and distinct.

Spectra taken in Region 5 showed some aspects similar to the spectra seen for Region 3. As shown in Figure 7 . Filament 3's spectrum showed faint emission from [N II] $6583 \AA$, and [S II] 6716, $6731 \AA$ like that seen for Filament 2 in Region 3 but now with no [N I] or [O I] emission. Notably however, the easternmost edge of this filament, covering roughly the first $2^{\prime \prime}$ along the slit, showed only $\mathrm{H} \alpha$ and $\mathrm{H} \beta$ emission lines.

Emission from extended nebulosity farther to the east (Filament 4) showed stronger [N II] emission along with the additional presence of [O III] $5007 \AA$ line emission. The appearance of [O III] emission started abruptly roughly $10^{\prime \prime}$ east of Filament 3 and extended some $20^{\prime \prime}$ along the slit to the east.

The measured $\mathrm{H} \alpha / \mathrm{H} \beta$ ratios for Filament 2 in Region 3 and Filament 3 in Region 5 are 3.14 and 3.4, respectively. Assuming an intrinsic $\mathrm{H} \alpha / \mathrm{H} \beta$ of 2.87 under the assumption of large optical depths in the Lyman series (i.e., Case B; see Table 4.2 in Osterbrock \& Ferland 2006) and an electron temperature of $10^{4} \mathrm{~K}$, we find a $\mathrm{E}(\mathrm{B}-\mathrm{V})$ values of $0.085 \pm 0.02 \mathrm{mag}$ and $0.15 \pm 0.05$ mag for Filaments 2 and 3 , respectively. Due to the faintness of Filament 3 of Region 5 compared to Filament 2 in Region 3, we have given more weight to its $\mathrm{E}(\mathrm{B}-\mathrm{V})$ value and estimate an $\mathrm{E}(\mathrm{B}-\mathrm{V})$ value for $\mathrm{G} 70$ of $0.10 \pm 0.3$.

This value is close to the $\mathrm{E}(\mathrm{B}-\mathrm{V})$ value of 0.08 seen for the neighboring Cygnus Loop SNR (Parker 1967; Fesen et al. 1982). This small amount of reddening to the G70 filaments is not surprising given their location so far off the galactic plane.

The electron density sensitive [S II] 6716/6731 emission line ratio of $1.3 \pm 0.15$ for Filament 2 indicates a density $\left(\mathrm{n}_{\mathrm{e}} \leq 200 \mathrm{~cm}^{-3}\right)$ and close to the low density limit of the $[\overline{\mathrm{S}} \mathrm{II}]$ line ratio. Electron densities at or near the low density [S II] ratio limit of 1.4 is typical for SNRs (Fesen et al. 1985). The $\mathrm{S} / \mathrm{N}$ of the other spectra where [S II] line emission is seen is too low to give reliable density estimates.

\subsection{Shock Emission Filaments}

Balmer dominated emission filaments like those seen for Filament 1 have been seen in a few Galactic SNRs and are thought to be the result of a high velocity shock moving through a partially neutral medium producing Balmer dominated emission filaments (Chevalier et al. 1980). The $\mathrm{H} \alpha$ emission line profile for such filaments consists of two components; a narrow component generated by the post-shock electron excitation of cold 


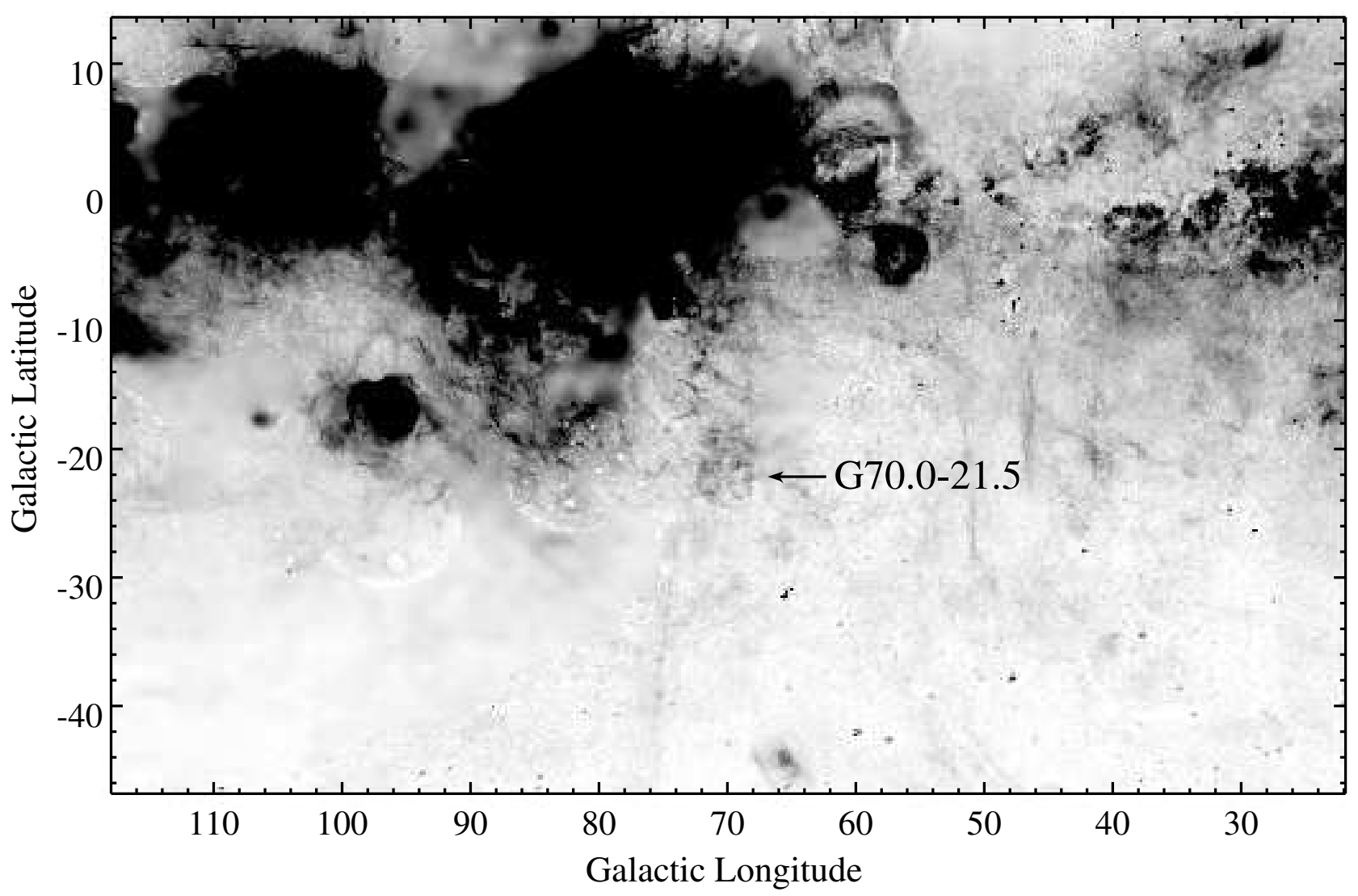

FIG. 1.- $\mathrm{H} \alpha$ image of the G70.0-21.5 nebulosity as seen on the Virginia Tech Spectral Line Survey (VTSS) of the Galactic Plane.

preshock neutral hydrogen atoms passing through a collisionless shock front, and a much broader component created via charge exchange between neutral hydrogen atoms and hot postshock protons.

Such Balmer filaments are characteristically thin and mark the current location of a remnant's advancing interstellar shock front. They are sometimes referred to as nonradiative filaments to distinguish them from the typically brighter, cooling radiative shock filaments commonly seen in SNRs. Examples of Balmer dominated filaments include the optical filaments in the Tycho and SN 1006 remnants, portions of the Kepler and RCW 86 remnants, and an array of faint, thin outlying filaments along the eastern and northern boundaries of the Cygnus Loop (see Heng 2010 for a review).

In young remnants like Tycho, Kepler, and SN 1006, the estimated shock velocity responsible for Balmer dominated filaments is $\simeq 1500-2000 \mathrm{~km} \mathrm{~s}^{-1}$. However, much lower shock velocities in the range of $150-450 \mathrm{~km} \mathrm{~s}^{-1}$ have been estimated for Balmer dominated filaments in the Cygnus Loop (Hester et al. 1994; Medina et al. 2014) and in G159.6+7.3 (Fesen \& Milisavlievid 2010).

The optical filaments seen in G70 are similar in morphology to Balmer filaments in the the Cygnus Loop (Hester et al.|1994; Medina et al. 2014) and G156.2+5.7 (Gerardy \& Fesen 2007). Specifically, G70 filaments seen in Regions 1, 2 (Fig. 3) and parts of Region 3 appear strikingly similar to Balmer filaments seen along the outer northeastern limb of the Cygnus Loop.

While the spectrum for Filament 1 indicates a Balmer dominated shock emission, the presence of other emission lines in the spectrum for Filament 2 are unusual for Balmer dominated filaments. Faint [N II] and [S II] line emissions have been seen in some Balmer filaments in the Cygnus Loop (Fesen \& Itoh 1985), Kepler's SNR (Blair et al. 1991), and Tycho's SNR (Ghavamian et al. 2000) and have been attributed to shock precursor emissions ionizing the preshock material located out ahead of the shock front (Hester et al. 1994; Ghavamian et al. 2000).

Some other Balmer dominated filaments show weak [O III] $5007 \AA$ line emission. For example, in the case of Cygnus Loop Balmer filaments, [O III] $5007 \AA$ has been observed with a strength around $10 \%$ that of $\mathrm{H} \beta$ and can be even stronger in cases where the shocked filament is undergoing a transition from a nonradiative adiabatic shock to one that is radiative (Hester et al. 1994). Our detection of [O III] emission for Filament 4 is of similar strength relative $\mathrm{H} \alpha$ to these other cases.

However, the presence of [N I] 5198, $5200 \AA$, [O I] 6300, $6364 \AA$ line emissions like seen in the Region 3 and 5 spectra has never been reported in any Balmer dominated filament spectrum but are common in radiative SNR filaments. Although weak [N I] and [O I] emissions could be the result of shock precursor emission, we suspect it is more likely to be due to a Balmer dominated filament transitioning from nonradiative to radiative. The presence of [O III] emission positioned some distance behind the leading edge of Region 5's filament fits with the picture of gas transitioning from nonradiative to radiative 


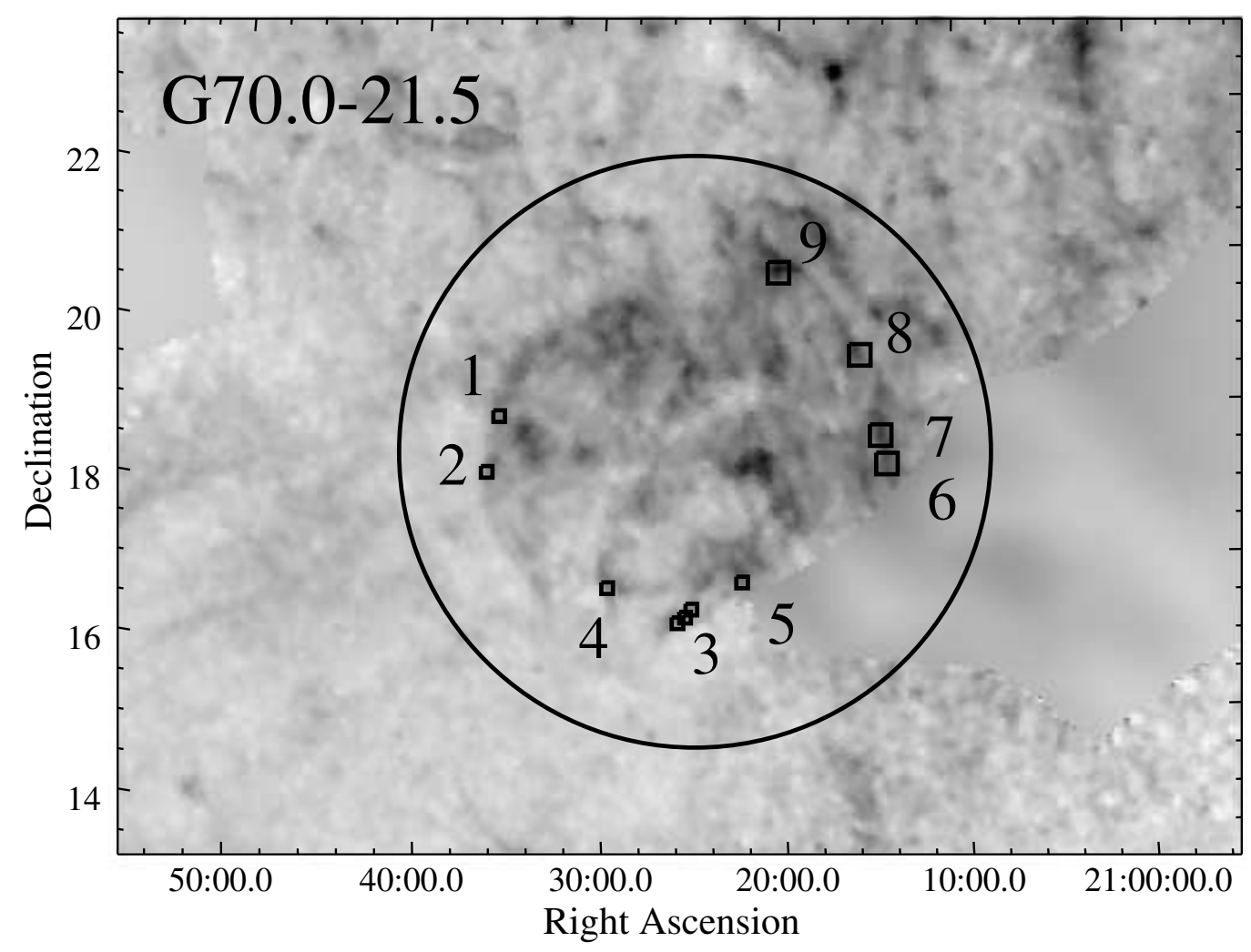

FIG. 2.- H $\alpha$ image of G70.0-21.5 from the Virginia Tech Spectral Line Survey (VTSS) of the Galactic Plane. North is up, East to the left with J2000 coordinates shown. The circle encloses the suspected extent of G70.0-21.5 with the numbered boxes marking the approximate regions $(1-9)$ where we obtained higher resolution $\mathrm{H} \alpha$ images. Low dispersion spectra were taken in Regions 3 and 5; see Fig. 6

emission.

Shocks below $90 \mathrm{~km} \mathrm{~s}^{-1}$ are predicted to exhibit strong line emissions from neutral and singly ionized species such as [N I], [N II], [S II] and [O I] with weak or absent [O III] 5007 emission (Shull \& McKee 1979; Ravmond 1979; Dopita et al. 1984). Thus, a Balmer dominated spectrum plus a contribution from a radiative $75-90$ $\mathrm{km} \mathrm{s}^{-1}$ shock like that in Model C of Shull \& McKee (1979) could generate a spectrum consistent with that observed for Regions 3 and 5. Support for this scenario is the presence of relatively strong [O I] 6300, $6364 \AA$ line emission ([O I] $6300 / \mathrm{H} \alpha>0.1$ ) which is commonly seen in SNRs and is characteristic of radiative shock emission (Fesen et al. 1985).

Additional evidence for shock velocities below $100 \mathrm{~km}$ $\mathrm{s}^{-1}$ for G70 filaments comes from the detection of faint near UV emission seen on GALEX images. As shown in Figure 8, several of G70's brighter filaments exhibit emission within GALEX's 1770 - $2730 \AA$ near UV bandpass presumably due to filament C III] $1909 \AA$ line emission. Far UV GALEX images $(1350-1780 \AA)$ covering these same filaments show only exceedingly weak emission presumably from C IV $1550 \AA$ A. Shock models predict weaker C IV than C III in shocks $\leq 90 \mathrm{~km} \mathrm{~s}^{-1}$ (Shull \& McKee 1979; Raymond 1979). Thus, a large C III] 1909/C IV 1550 emission ratio is consistent with a relatively low shock velocity as suggested by the presence of the [N I] and [O I] emissions seen in Filament 2.

\subsection{G70.0-21.5: A New Galactic Supernova Remnant}

We propose that the large G70.0-21.5 emission shell is a previously unrecognized Galactic supernova remnant. Both the morphology and spectra of G70.0-21.5's optical filaments are consistent with Balmer dominated shock emission transitioning into radiative filaments. The presence of diffuse, extended X-ray emission coincident with the G70 nebula lends further support to a supernova remnant identification.

Figure 9 shows VTSS $\mathrm{H} \alpha$ and ROSAT X-ray images of the G70 region. A faint, diffuse, crescent shaped Xray emission nebula can be seen to be coincident with the portion of the $\mathrm{H} \alpha$ nebulosity closest to the Galactic plane as shown in the optical and X-ray overlay shown in Figure 10. The presence of this isolated patch of X-ray emission situated far off the galactic plane and coincident with G70's location is consistent with a SNR nature.

The location of this X-ray emission projected inside the remnant's optical emission boundaries is also consistent with that of an evolved SNR where the X-ray emission appears centrally filled (Shelton 1999; Williams et al. 2004). Although the X-ray flux from this emission patch is too weak to produce a meaningful X-ray spectrum, its positional coincidence, size, and alignment with G70's northern shell lends additional support to G70's SNR identification.

The lack of reported nonthermal radio emission at G70's location (Shimmins \& Dav 1968; Green 2014a) is not surprising given the nebula's location of more than 21 degrees off the Galactic plane and thus outside the galactic latitude range usually searched for nonthermal radio emission (e.g., the Canadian Galactic Plane Survey; Tavlor et al. 2003). Of the 294 Galactic SNRs cataloged, 

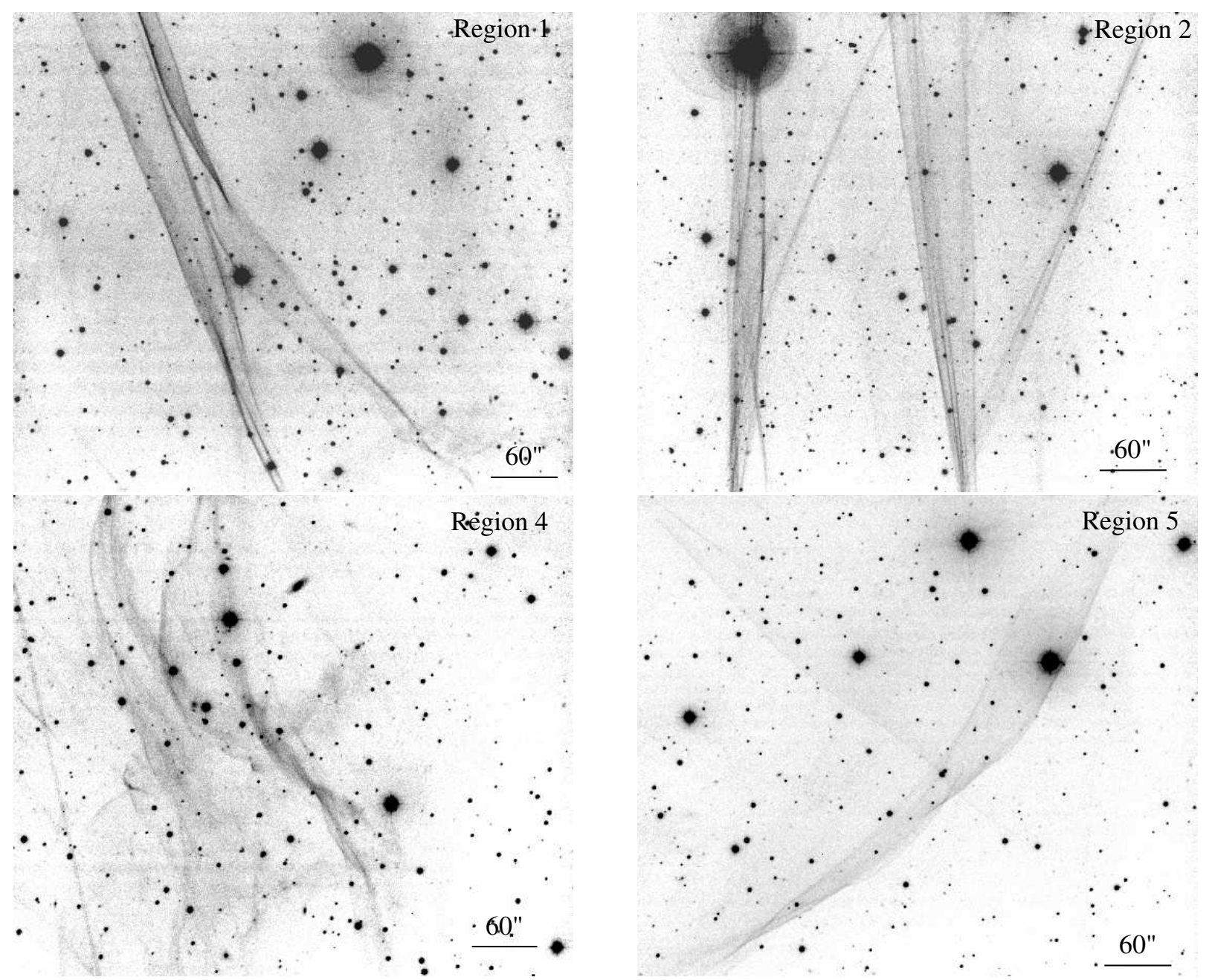

FIG. 3.- $\mathrm{H} \alpha$ images of Regions 1, 2, 4, and 5, in G70.0-21.5. North is up, East to the left.

G70.0-21.5 lies the furthest off the plane of the Galaxy, some 6 degrees farther than the current record-holder, the Lupus Loop (G330.0+15.0), and over 12 degrees farther off the plane than any SNR in the first quadrant (Green 2014b).

Although it does lie within the regions covered in galactic H I surveys aimed at identifying large shell-like H I structures, no H I shell possibly associated with G70 has been reported (Ehlerová \& Palouš 2013; Sallmen et al. 2015). This may be due in part to such H I surveys being particularly sensitive to large, low velocity old ISM shells with typical ages greater than several $10^{6}$ yrs (Ehlerová \& Palouš 2013), and thus much older than G70 and other large but optical and X-ray emitting SNRs.

With a diameter of roughly $4.0^{\circ} \times 5.5^{\circ}$, G70 ranks among the largest galactic SNRs known in terms of angular size. Because of its size and the presence of diffuse emission patches along its western limb, it is not known whether it is still in the Sedov-Taylor adiabatic expansion phase or has entered the later radiative expansion phase. Consequently, many of its basic physical parameters (age, distance, shock velocity, local ISM density) are uncertain. We can, however, place rough upper limits to its maximum distance based on arguments regarding its likely maximum physical size and z-distance off the galactic plane.

Based on a shock velocity estimate from our optical spectra, distances around $1-2 \mathrm{kpc}$ are likely from comparisons with other large remnants. At a distance of only $1 \mathrm{kpc}, \mathrm{G} 70$ 's linear dimensions would be $70 \times 95 \mathrm{pc}$, already larger than almost all known SNRs. Conversely, at distances less than $1 \mathrm{kpc}, \mathrm{G} 70$ would be among the closest remnants. Compared to the Cygnus Loop which has an estimated shock velocity of $150-350 \mathrm{~km} \mathrm{~s}^{-1}$ and diameter of $30-60$ pc (Blair et al. 2009; Medina et al. 2014) and the Monoceros Loop's $\mathrm{v}=50 \mathrm{~km} \mathrm{~s}^{-1}$ (dia. $\simeq 100$ pc; Leahy et al. 1986; Odegard 1986), a velocity $\simeq 75 \mathrm{~km} \mathrm{~s}^{-1}$ for $\mathrm{G} 70$ suggests it is less evolved than Monoceros but older than the Cygnus Loop and hence of a size intermediate to these.

G70 is unlikely to lie at distances much greater than $3 \mathrm{kpc}$ in order to have a z-distance less than the $500 \mathrm{pc}$ scale-height of the local H I halo including the Local Spur (Lockman et al. 1986; Cersosimo et al. 2009) and the zdistance of most metal poor stars (Bovy et al. 2012). Conversely, distances around $1.0 \mathrm{kpc}$ or less raises the possibility of detecting motion of G70's sharp filaments.

As mentioned in $\S 3.1$, some of G70's filaments are visible on the red Palomar Sky Survey images and we examined and compared our 2014 images with the digitized 1954 and 1991 POSS images (DSS1 and DSS2) covering 

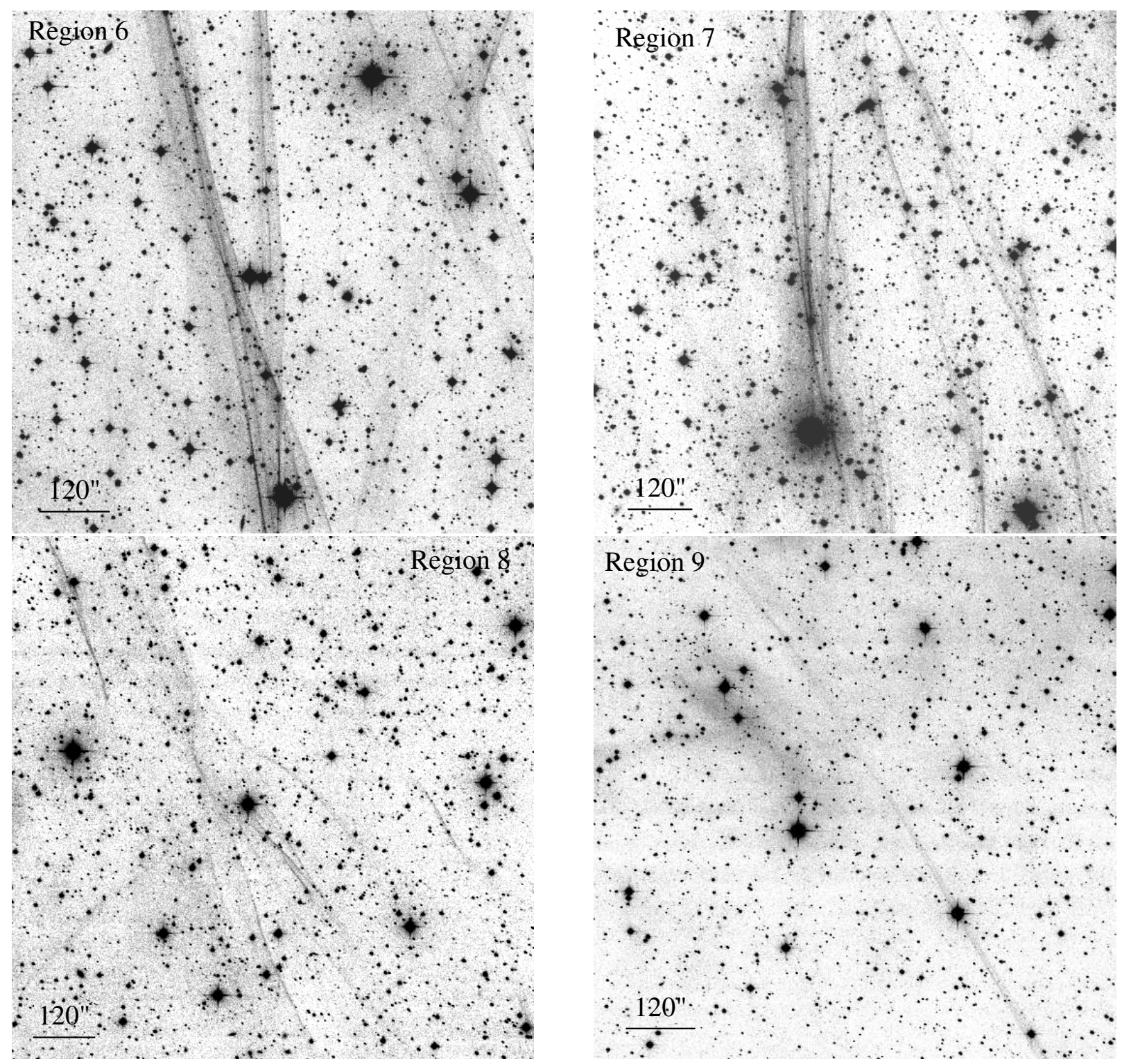

FIG. 4.- H $\alpha$ images of Regions 6, 7, 8 and 9 in G70.0-21.5. North is up, East to the left.

the G70 region. G70 filaments were too weakly detected on the DSS1 images to be useful, but comparisons of our 2014 and the DSS2 1991 images for G70's brighter filaments indicated proper motions in the range $00^{\prime \prime} 03-0 . \prime 06$ $\mathrm{yr}^{-1}$. Although these values have significant measurement uncertainties due to the very weak detection of the filaments on the broadband DSS2 images, such proper motions are consistent with a distance around $1 \mathrm{kpc}$ assuming a shock velocity $\simeq 75 \mathrm{~km} \mathrm{~s}^{-1}$.

Lastly, follow-up studies of this apparent SNR should prove fruitful. Detection of nonthermal radio emission from G70 could firmly establish its SNR nature. However, its radio emission might be quite weak, as SNR evolutionary models predict a rapid drop in radio emission from large remnants (Asvarov 2006). Furthermore, observations of extragalactic SNRs suggest especially weak radio emission for SNRs expanding in low density media (Pannuti et al. 2002). Nonetheless, existing or future galactic radio surveys (e.g., the Arecibo G-ALFA Transit Survey; Taylor \& Salter 2010) might detect nonthermal emission from G70.

The probable low ambient density at G70's high zdistance is likely the cause for its optical emission to be nearly entirely Balmer dominated filaments, a property rare in SNRs. Although faint, the presence of so many Balmer dominated filaments throughout G70 and the variation of emission line strength we observed across some of its filaments may make it a valuable remnant for better understanding the transition of nonradiative to radiative shocks. Finally, investigation of its X-ray emission could provide information regarding its shock velocity and age.

In summary, we believe the nebulosity, G70.0-21.5, to be a physically large and relatively old SNR that lies unusually high off the galactic plane. It exhibits a rich array of beautiful and overlapping Balmer dominated filaments covering a region over 4 degrees in size. At a likely distance around 1 to $2 \mathrm{kpc}, \mathrm{G} 70.0-21.5$ also ranks among the closest remnants known.

We thank an anonymous referee for comments and a 


\section{Region 3}
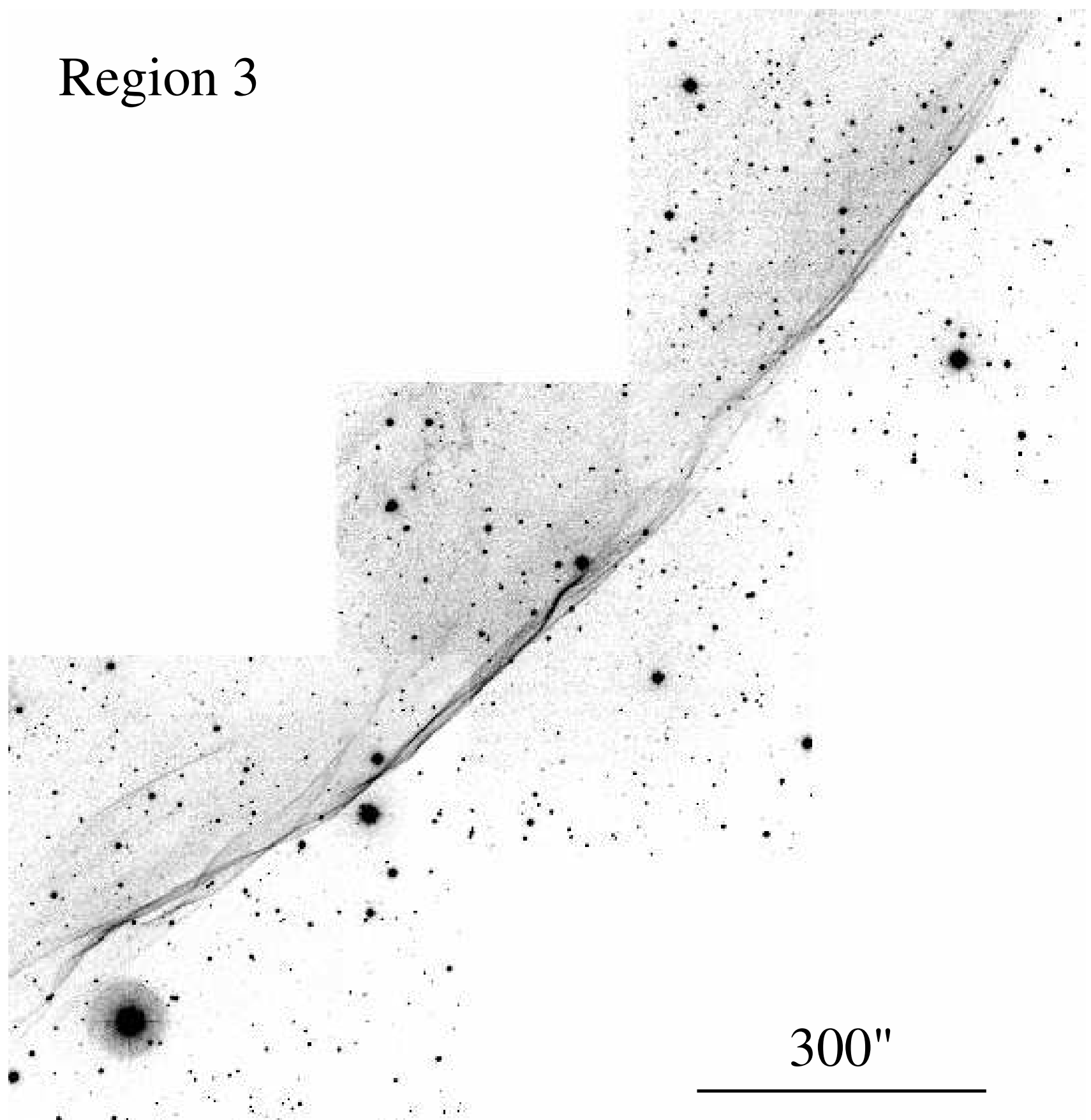

Fig. 5.- Mosaic of $\mathrm{H} \alpha$ images covering Region 3 in G70.0-21.5. North is up, East to the left.

presentation, and D. Patnaude and D. Milisavljevic for helpful discussions.

\section{REFERENCES}

Asvarov, A. I. 2006, A\&A, 459, 519

Blair, W. P., Sankrit, R., Torres, S. I., Chayer, P., \& Danforth, C. W. 2009, ApJ, 692, 335

Blair, W. P., Long, K. S., \& Vancura, O. 1991, ApJ, 366, 484

Bovy, J., Rix, H.-W., \& Hogg, D. W. 2012, ApJ, 751, 131

Chevalier, R. A., Kirshner, R. P., \& Raymond, J. C. 1980, ApJ, 235,186

Cersosimo, J. C., Muller, R. J., \& Figueroa, N. S. 2009, ApJ, 699, 716

de Gasperin, F., Evoli, C., Brüggen, M., et al. 2014, A\&A, 568, A107
Dennison, B., Simonetti, J. H., \& Topasna, G. A. 1998, Publications of the Astronomical Society of Australia, 15, 147 Dopita, M. A., Binette, L., Dodorico, S., \& Benvenuti, P. 1984, ApJ, 276, 653

Downes, D. 1971, AJ, 76, 305

Drew, J. E., Greimel, R., Irwin, M. J., et al. 2005, MNRAS, 362, 753

Ehlerová, S., \& Palouš, J. 2013, A\&A, 550, A23

Fesen, R. A., Blair, W. P., \& Kirshner, R. P. 1982, ApJ, 262, 171

Fesen, R. A., Blair, W. P., \& Kirshner, R. P. 1985, ApJ, 292, 29

Fesen, R. A., \& Itoh, H. 1985, ApJ, 295, 43 
TABLE 1

IMAGED REGions OF G70.0-21.5

\begin{tabular}{|c|c|c|c|c|c|}
\hline Region & $\begin{array}{c}\text { RA }(\mathrm{J} 2000) \\
\mathrm{h} \text { m s }\end{array}$ & $\operatorname{Dec}\left(\mathrm{J}_{\prime} 2000\right)$ & ${ }^{l}$, & $\circ$ & $\begin{array}{c}\text { Exposures } \\
\text { (s) }\end{array}$ \\
\hline 1 & $21: 35: 18$ & $+19: 19: 54$ & $71: 55: 03$ & $-23: 33: 17$ & $3 \times 600$ \\
\hline 2 & $21: 35: 56$ & $+18: 36: 04$ & $71: 26: 32$ & $-24: 09: 40$ & $3 \times 600$ \\
\hline 3 & $21: 25: 08$ & $+16: 57: 07$ & $68: 13: 46$ & $-23: 20: 08$ & $2 \times 600$ \\
\hline 4 & $21: 29: 21$ & $+17: 15: 26$ & $69: 12: 08$ & $-23: 53: 49$ & $2 \times 600$ \\
\hline 5 & $21: 21: 59$ & $+17: 26: 56$ & $68: 06: 40$ & $-22: 26: 30$ & $2 \times 600$ \\
\hline 6 & $21: 13: 51$ & $+19: 04: 38$ & 68:07:04 & $-19: 55: 01$ & $3 \times 600$ \\
\hline 7 & $21: 14: 10$ & $+19: 26: 37$ & $68: 28: 05$ & $-19: 44: 20$ & $3 \times 900$ \\
\hline 8 & $21: 15: 16$ & $+20: 28: 34$ & 69:29:00 & $-19: 16: 23$ & $1 \times 900$ \\
\hline 9 & $21: 19: 50$ & $+21: 29: 09$ & $71: 01: 50$ & $-19: 46: 31$ & $2 \times 900$ \\
\hline
\end{tabular}

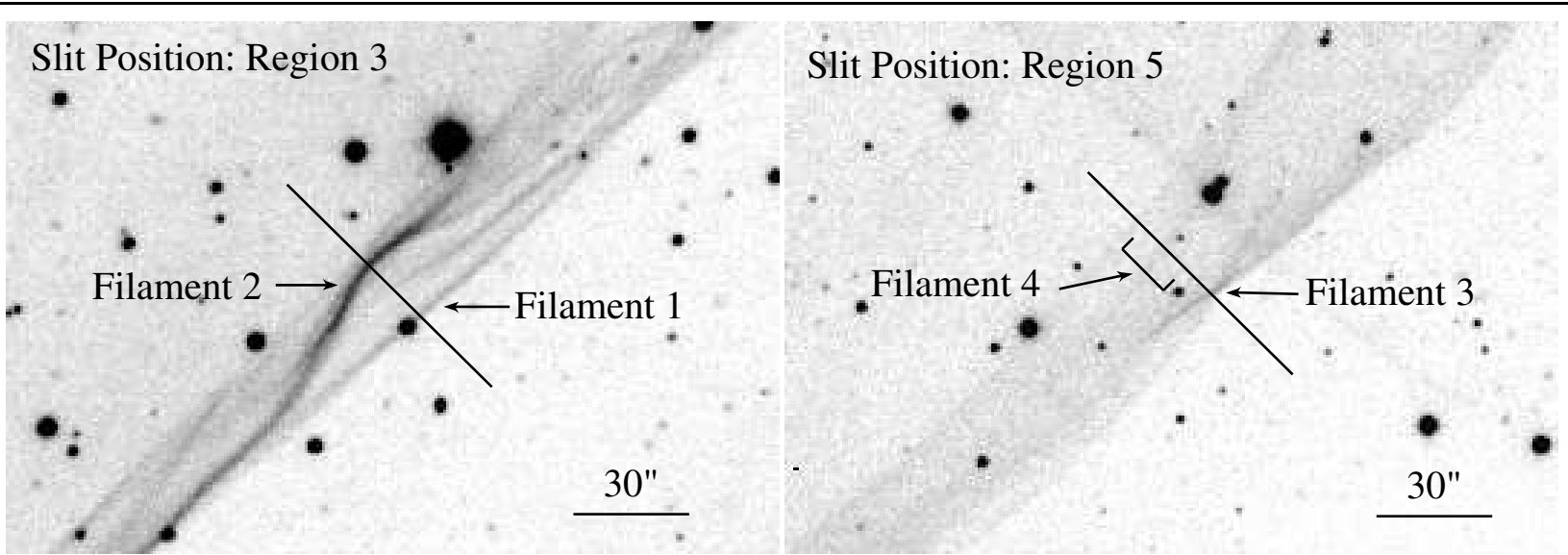

Fig. 6.- Slit locations on $\mathrm{H} \alpha$ images of Regions 3 and 5 in G70.0-21.5 where spectra where taken. North is up, East to the left.

Fesen, R. A., \& Milisavljevic, D. 2010, AJ, 140, 1163

Finkbeiner, D. P. 2003, ApJS, 146, 407

Gao, X. Y., \& Han, J. L. 2014, A\&A, 567, A59

Gerardy, C. L., \& Fesen, R. A. 2007, MNRAS, 376, 929

Gerbrandt, S., Foster, T. J., Kothes, R., Geisbüsch, J., \& Tung, A. 2014, A\&A, 566, 76

Ghavamian, P., Raymond, J., Hartigan, P., \& Blair, W. P. 2000, ApJ, 535, 266

González-Solares, E. A., Walton, N. A., Greimel, R., et al. 2008, MNRAS, 388, 89

Green, D. A. 2014, Bulletin of the Astronomical Society of India, 42,47

Green, D. A. 2014, IAU Symposium, 296, 188

Gull, T. R., Kirshner, R. P., \& Parker, R. A. R. 1977, ApJ, 215 L69

Heng, K. 2010, Pub. Astro. Soc. Pacific, 27, 23

Hester, J. J., Raymond, J. C., \& Blair, W. P. 1994, ApJ, 420, 721

Lockman, F. J., Hobbs, L. M., \& Shull, J. M. 1986, ApJ, 301, 380

Leahy, D. A., Naranan, S., \& Singh, K. P. 1986, MNRAS, 220, 501

Maršálková, P. 1974, Ap\&SS, 27, 3

Massey, P., \& Gronwald, C. 1990, ApJ, 358, 344

Medina, A. A., Raymond, J. C., Edgar, R. J., et al. 2014, ApJ, 791, 30

Milne, D. K. 1970, Australian Journal of Physics, 23, 425

Neckel, T., \& Vehrenberg, H. 1985, Atlas of Galactic Nebulae, Vol. I \& II, Duesseldorf: Treugesell-Verlag, 1985

Odegard, N. 1986, ApJ, 301, 813

Oke, J. B. 1974, ApJS, 27, 21

Osterbrock, D. E., \& Ferland, G. J. 2006, Astrophysics of gaseous nebulae and active galactic nuclei, 2nd. ed. Sausalito, CA: University Science Books
Pannuti, T. G., Duric, N., Lacey, C. K., Ferguson, A. M. N., Magnor, M. A., \& Mendelowitz, C. 2002, ApJ, 565, 966

Parker, R. A. R. 1967, ApJ, 149, 363

Parker, R. A. R., Gull, T. R., \& Kirshner, R. P. 1979, NASA SP-434

Raymond, J. C. 1979, ApJS, 39, 1

Raymond, J. C., Davis, M., Gull, T. R., \& Parker, R. A. R. 1980, ApJ, 238, L21

Renaud, M., Marandon, V., Gotthelf, E. V., et al. 2010, ApJ, 716, 663

Reynolds M. T., et al., 2013, ApJ, 766, 112

Sabin, L., Parker, Q. A., Contreras, M. E., et al. 2013, MNRAS, 431, 279

Sallmen, S. M., Korpela, E. J., Bellehumeur, B., et al. 2015, AJ, 149,189

Sankrit, R., Blair, W. P., Delaney, T., et al. 2005, Advances in Space Research, 35, 1027

Sharpless, S. 1959, ApJS, 4, 257

Shimmins, A. J., \& Day, G. A. 1968, Australian Journal of Physics, 21, 377

Shelton, R. L. 1999, ApJ, 521, 217

Shull, J. M., \& McKee, C. F. 1979, ApJ, 227, 131

Sivan, J. P. 1974, A\&AS, 16, 163

Stupar, M., Parker, Q. A., \& Filipović, M. D. 2008, MNRAS, 390, 1037

Taylor, A. R., Gibson, S. J., Peracaula, M., et al. 2003, AJ, 125, 3145

Taylor, A. R., \& Salter, C. J. 2010, The Dynamic Interstellar Medium: A Celebration of the Canadian Galactic Plane Survey, 438, 402

Williams, R. M., Chu, Y.-H., Dickel, J. R., Gruendl, R. A., Shelton, R., Points, S. D., \& Smith, R. C. 2004, ApJ, 613, 948 


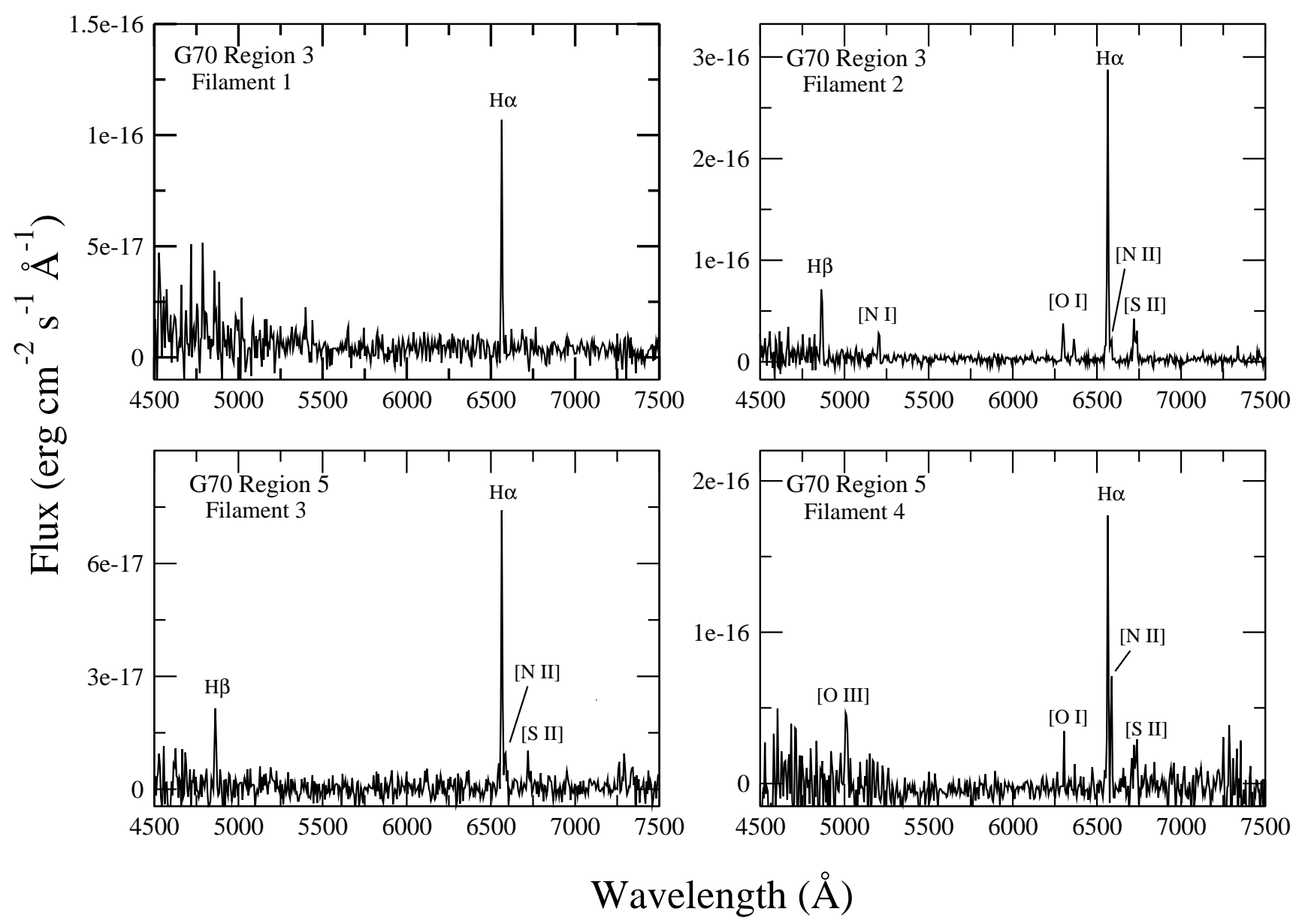

Fig. 7.- Spectra of emission filaments in Regions 3 and 5. 


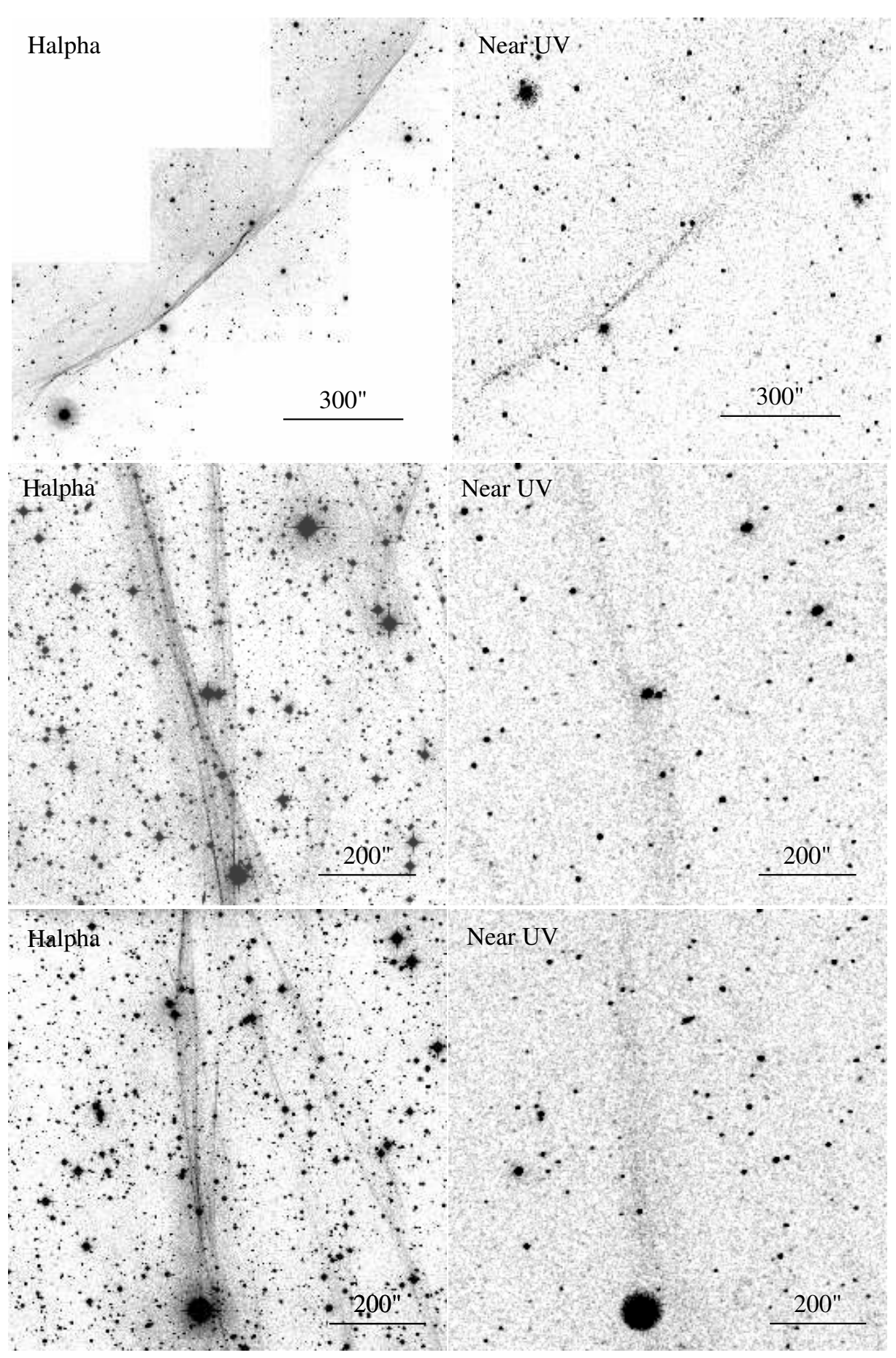

FIG. 8. - Comparison of $\mathrm{H} \alpha$ and GALEX near UV emission (1770 - $2730 \AA$ ) images for Regions 3, 6 , and 7 of G70.0 - 21.5 in the top, middle, and bottom panels, respectively. 

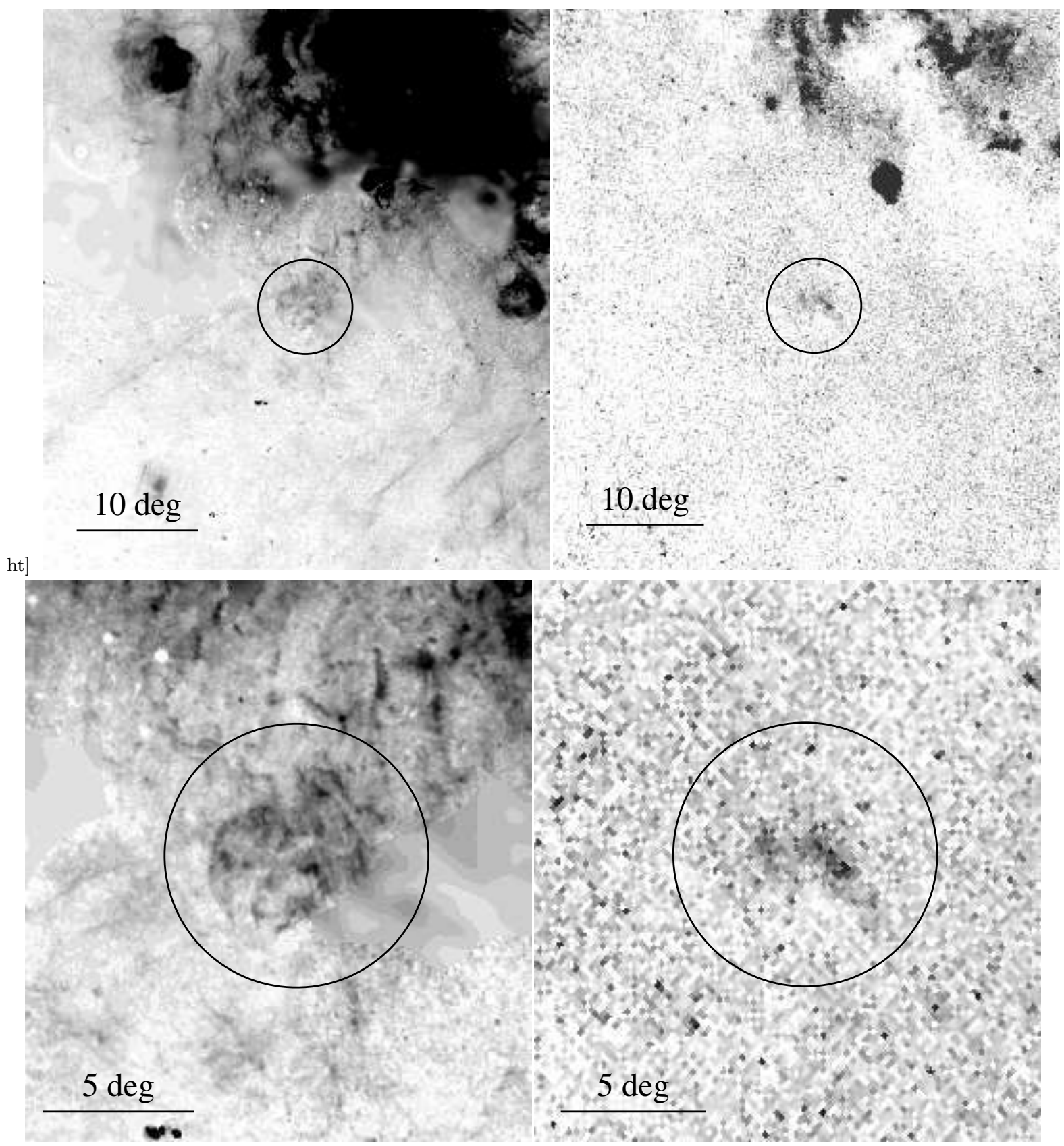

FIG. 9.- Upper panels show the VTSS H $\alpha$ image (left) and the ROSAT All Sky Background Survey (RASS3) X-ray image (right) of a large region off the Galactic plane centered on G70.0-21.5 (marked by the circles) illustrating the lack of X-ray emission off the galactic plane around G70.0-21.5. North is up, East to the left. The remnant's associated X-ray emission is curved and lies on the remnant's side closest to the Galactic plane. The bright elliptical X-ray source 12 degrees northwest of G70.0-21.5 is the Cygnus Loop SNR. Lower panels show enlargements of these $\mathrm{H} \alpha$ and X-ray images. 


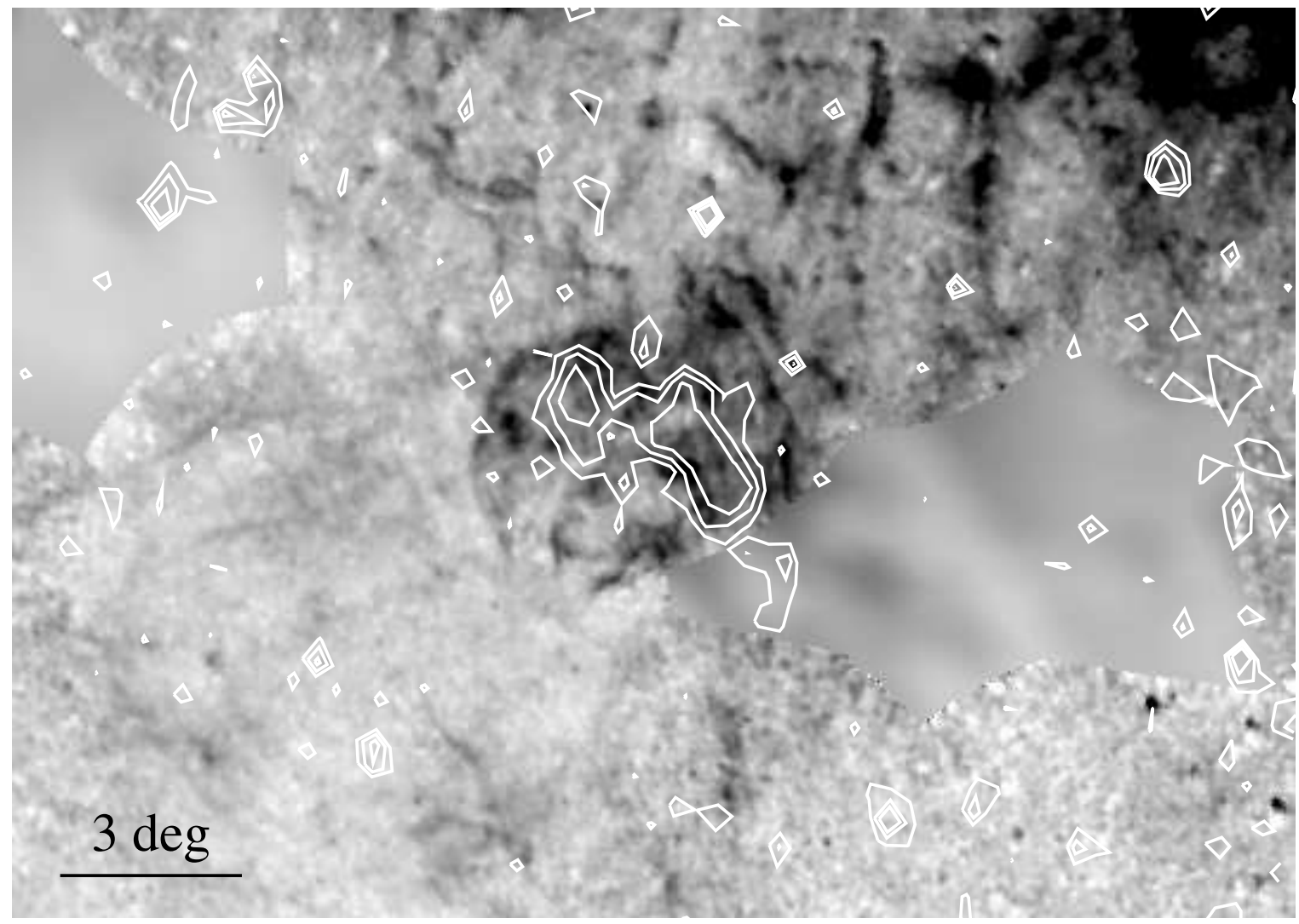

FIG. 10.- X-ray emission contours from the ROSAT All Sky Background Survey (RASS3) onto the VTSS H $\alpha$ image of G70.0-21.5. North is up, East to the left. Note that the detected X-ray emission lies within the projected boundaries of the remnant's optical emission. 